\title{
Gene Expression Profiling in Organ Transplantation
}

\author{
Osama Ashry Ahmed Gheith \\ Urology and Nephrology Center, Mansoura University, Mansoura, Egypt \\ Correspondence should be addressed to Osama Ashry Ahmed Gheith, ogheith@yahoo.com
}

Received 11 April 2011; Accepted 22 June 2011

Academic Editor: James E. Springate

Copyright (C) 2011 Osama Ashry Ahmed Gheith. This is an open access article distributed under the Creative Commons Attribution License, which permits unrestricted use, distribution, and reproduction in any medium, provided the original work is properly cited.

\begin{abstract}
Aim of Review. Huge effort is being made among the transplant community investigating novel biomarkers that enable transplant clinicians to identify patients at risk for allograft rejection or those who will develop tolerance so that immunosuppression could be safely minimized or even ideally withdrawn. Despite the important advances achieved in the identification of several potential biomarkers of tolerance, rejection, or both, validation and demonstration of their clinical utility still needs to be tested, which will need international cooperative networks. It is important to note that the reproducibility of differently expressed genes might be affected by many factors such as gene ranking and selection methods, inherent differences between types, and the choice of thresholds. However, because microarray analyses are expensive and time consuming and their statistical evaluation is often very difficult, gene expression analysis using the RTPCR method is nowadays recommended. Conclusions. In the field of organ transplantation, gene-expression-based decision might help in improving patient and graft outcome and there are a multitude of studies showing that gene-expression profiling is feasible.
\end{abstract}

\section{Introduction}

Despite the improvement in both patient and short-term renal allograft survival in the last decade, probably because of newer and more specific immunosuppressive agents in addition to improved surgical and medical cares, Longterm graft failure continues to plague kidney allografts with both immune-dependent and -independent factors which continue to contribute to failure. Even the morbidity and mortality remain much higher than in the general population, mainly due to the complications of immunosuppressive therapy. A number of promising observations made in human kidney recipients suggest unique protein and genetic signatures that may identify biomarkers of injury, as well as potential targets of therapy. Some of these may be obtained through noninvasive methods and may thus be extremely useful in the clinical realm $[1,2]$.

Nonspecific etiopathogenic graft parenchymal lesions in allograft biopsies, graft function evolution using surrogate markers of graft dysfunction such as serum creatinine or proteinuria, and indirect measurements of the alloimmune response using blood trough levels of immunosuppressants are the current markers used worldwide in the clinic in renal transplantation [3].

\section{Biomarkers of Allograft Outcome}

Huge effort is being made among the transplant community investigating novel biomarkers at different biological levels that enable transplant clinicians to identify patients at risk for allograft rejection or, conversely, patients in whom immunosuppression could be safely minimized or even ideally withdrawn because of their biological suitability to accept the renal allograft. The search for biomarkers of tolerance or rejection has been focusing at different biological levels, including analysis of sera looking for alloantibodies, peripheral blood mononuclear cells (PBMCs) using different techniques (flow cytometry, microarrays, and reverse transcription PCR), analysis of the urine at the protein, peptide or transcriptomic level, histological assessment of transplant biopsies, and, finally, functional assays measuring alloreactive or antiviral PBMC responses. Because of the relatively easy induction of permanent renal allograft acceptance in mice with minimal amount of immunosuppression, 
some groups have assessed the expression of certain genes during either rejection or permanent graft acceptance both in peripheral blood and within the graft tissue. Interestingly, the assessment of two particular gene fragments such as tolerance-associated gene-1, a mitochondrial protein T-cell apoptosis (TOAG-1), and a-1,2-mannosidase (relevant gene for the N-glycosylation of membrane bound and secreted proteins) in a model of kidney and cardiac rat transplantation was shown to be downregulated both in peripheral blood and within the kidney graft prior and during acute and chronic rejection and highly expressed during induction and maintenance of graft acceptance with high specificity and reproducibility [4].

\section{Ongoing Studies}

Both clinical and experimental observations suggest that allograft rejection is a complex process with multiple components that are, at least partially, functionally redundant. Studies using graft recipients which are deficient in certain genes-including chemokines, cytokines, and other immune-associated genes-frequently produce a phenotype of delayed, but not indefinitely prevented, rejection. Only a small subset of genetic deletions (e.g., TCR_ or -, MHC I and II, B7-1 and B7-2, and recombinase-activating gene) permit permanent graft acceptance suggesting that rejection is orchestrated by a complex network of interrelated inflammatory and immune responses. To investigate this complex process, oligonucleotide microarrays have been used to generate quantitative mRNA expression profiles following transplantation. Patterns of gene expression were confirmed with real-time PCR data. Hierarchical clustering algorithms clearly differentiated the early and late phases of rejection. Self-organizing maps identified clusters of coordinately regulated genes. Genes upregulated during the early phase included genes with prior biological functions associated with ischemia, injury, and Ag-independent innate immunity, whereas genes upregulated in the late phase were enriched for genes associated with adaptive immunity [5].

There are several stages to perform a microarray analysis [6]: preparation of the microarray; generation of fluorescent targets from the RNA of the samples, hybridization to the probes, data acquisition: scanning of the signal intensity emanating from the hybridized labeled probes, and then data analysis which is often the most challenging component of GEP. In general, cDNA microarray experiments require more than 50 micrograms of total RNA from target tissues, while 5 micrograms may be sufficient for an oligo array experiment. Moreover, in a cDNA microarray, a control sample (e.g., normal as compared to diseased tissue) is simultaneously analyzed along with the test sample on the same chip.

The development of high microarray technology-by assessing changes in expression of multiple genes and quantitation of proteins within human cells, plasma, or tissues-will provide the opportunity for novel insight into molecular pathways of tissue injury and disease processes [7-12]. Recently, the diagnostic sensitivity, specificity, and accuracy of histological diagnosis using this technology have been improved $[13,14]$ and both biomarker panels and individual biomarkers have been identified within the allograft to improve the diagnostic, prognostic, and potentially therapeutic categorization of acute rejection [15-18].

\section{Gene Expression: New Diagnostic Procedure in Organ Transplantation}

Noninvasive sources such as peripheral blood have increasingly been targeted to provide information in the field of organ transplantation [19-22].

Recipient gene expression is profoundly altered after graft implantation. Some of these changes are characteristic of rejection injury. The biological function of the expressed genes encompasses major biological categories of cellular processes related to immune signal transduction, cytoskeletal reorganization, and apoptosis and emphasizes the participation of the cytokine-activated Jak-Stat pathway and interferon signaling in lymphocyte activation proliferation, chemotaxis, and adhesion [23].

Cohen Freue et al. 2010, have observed that the characteristic changes in the plasma proteome were paralleled to plasma proteins changes that encompassed processes related to inflammation, complement activation, blood coagulation, and wound repair which were significantly different between patient cohorts with and without biopsy-confirmed acute rejection [24].

Martínez-Llordella et al., 2008 reported that measurement of the expression levels of a small set of genes in peripheral blood could be useful to accurately identify liver recipients who are able to accept their grafts in the absence of pharmacological immunosuppression. Validation of such findings in prospective immunosuppression weaning trials would open the door to the possibility of withdrawing immunosuppressive drugs in transplant recipients with high likelihood of being tolerant. Further, functional analysis of expression patterns suggests that molecular pathways involved in the activation and function of innate immunity cell types (NK and $\gamma \delta \mathrm{TCR}+\mathrm{T}$ cells) are central to the maintenance of operational tolerance following liver transplantation. All these findings explain the value of peripheral blood transcriptional profiling in the immune monitoring of liver transplant recipients and provide insight into the pathogenesis of human allograft tolerance [25].

In this direction, different effector, regulatory and cytotoxic gene cytokine expression, has also been analyzed. Different expression patterns of selected genes have been reported to be upregulated or downregulated during allograft rejection (both $\mathrm{T}$ cell or antibody mediated), in nonimmune-mediated allograft dysfunction or in stable functioning allografts. Preliminary reports, from two large consortia, showed that similar observations in tolerant patients were observed, in terms of increased expression of B-cell genes as compared with patients under conventional immunosuppression [26, 27]. Interestingly and in such direction, low expression of the mRNA B-cell marker CD20 has been observed within grafts that failed as a consequence of either T-cell or antibody-mediated rejection as compared 
with those that had a favorable course [28]. Furthermore, the Nantes group has shown increased mRNA levels of two interesting markers (granzyme B and Tribbles-1) that were assessed in peripheral blood and in graft tissue in patients undergoing chronic antibody-mediated rejection as compared with patients with a nonimmune-mediated chronic graft dysfunction $[29,30]$.

Matsui et al. [31] identified two genes (H2-Ea and Frzb) that were highly expressed in long-term surviving heart allografts. Moreover, another group has recently reported an interesting upregulation of specific set of genes (TGFb2, ppENK, GM2a, GITR, and IL-1R2) in regulatory T cells (Tregs) associated with tolerance of skin allografts [32, 33].

Some reports have assessed the gene expression profile (GEP) in different settings during renal transplantation. The use of DNA microarrays, which allow detailed measurements of gene expression in a global scale, both in graft tissue samples and in peripheral blood, has been extensively used. Sarwal et al. [34] investigated the molecular basis of acute rejection analyzing the gene expression patterns in 67 allograft biopsies using DNA microarrays. Interestingly, extensive differences in the gene expression in patients with the same histological pattern of acute rejection were observed. Such differences were associated with different immunologic and cellular features as well as with a different clinical outcome.

The molecular characterization using Affymetrix DNA microarrays (http://affymetrix.com) has been assessed by Flechner et al. [35], using both peripheral blood and graft tissue samples at the same time. They were able to show a unique expression signature with upregulation of certain immune/inflammatory and profibrotic/fibroticassociated genes in patients with high Banff score lesions, in patients under calcineurin inhibitors (CNI), and in patients with well-functioning transplant allografts.

\section{Clinical Applications in Organ Transplantation Field}

In the same direction, Mas et al. [36], using Affymetrix microarrays in 17 renal tissue samples from 18 renal transplant patients, identified a 728-probe set genes related to immune response (cytokine, chemokine, and receptor genes) and to fibrosis and extracellular matrix deposition, differentially expressed between 11 kidneys with histological proven lesions of chronic rejection and seven transplant controls.

On the other hand, Brouard et al. [37] reported a potential tolerance gene signature using pan-genomic cDNA microarrays and real-time (RT)-PCR in PBMCs. Interestingly, they were able to screen 17 kidney transplant patients with operational tolerance and compared with patients undergoing acute or chronic rejection, others with well-functioning grafts and healthy volunteers. A tolerance "footprint" of 49 genes was able to correctly separate the tolerance and chronic rejection phenotypes with high specificity (100\%) and sensitivity (90\%). Also, they could identify this gene signature in six of 22 patients under immunosuppression. Importantly, none of the eight healthy age-matched controls showed the "tolerant fingerprint," suggesting that it is not only a gene signature for being off of immunosuppression. Expression of costimulatory genes and markers of early and late T-cell activation was reduced in tolerant patients and many transforming growthfactor-beta-regulated genes were interestingly upregulated. Similarly, but in the liver transplant setting, MartínezLlordella et al. [38], using microarray analyses followed by RT-PCR, were able to describe a tolerance gene expression signature in an initial set of 17 tolerant and 21 nontolerant liver transplant recipients initially containing 68 genes, which comprised a large number of transcripts associated with natural killer and gdT cells.

Among lung transplant recipients, Xu et al. 2005, utilized this highly sensitive method using small, bronchoscopically acquired biopsy specimens of allografts. Transcript signatures obtained by this approach suggested that airway and alveolar responses to rejection differ and that endobronchial biopsy specimens assess lymphocytic bronchitis and chronic rejection but are not proxies for transbronchial biopsy specimens. Moreover, they reveal changes in airway expression of the specific genes involved in host defense and remodeling and suggested that measurement of transcripts correlating with lymphocytic bronchitis may be diagnostic adjuncts to histopathology [39]. Deng et al. 2006 [40] reported that gene expression testing can detect absence of moderate/severe rejection-although this need additional clinical experience-thus avoiding biopsy in certain clinical settings as cardiac transplant recipients.

Bodonyi-Kovacs et al. 2010 [41], had demonstrated that biomarkers of inflammation and immune activity detected within intraoperative renal transplant allograft biopsies are linked to adverse short-term posttransplantation clinical outcomes. Moreover, they theorized that targeted polymerase chain reaction profiling of gene expression in the donor kidney at the time of engraftment can predict 2-year post-transplantation clinical outcomes.

It is important to note that the reproducibility of differently expressed genes might be affected by many factors such as gene ranking and selection methods, inherent differences between types, and the choice of thresholds. However, because microarray analyses are expensive and time-consuming and their statistical evaluation is often very difficult, gene expression analysis using the RTPCR method is nowadays recommended [42].

\section{Conclusions}

In the field of organ transplantation, gene-expression-based decision might help in improving patient and graft outcome and there are a multitude of studies showing that geneexpression profiling is feasible.

\section{References}

[1] O. Bestard, J. M. Cruzado, M. L. Franquesa, and J. M. Grinyó, "Biomarkers in renal transplantation," Current Opinion in Organ Transplantation, vol. 15, no. 4, pp. 467-473, 2010. 
[2] R. B. Mannon, "Immune monitoring and biomarkers to predict chronic allograft dysfunction," Kidney International, vol. 78, no. 119, pp. S59-S65, 2010.

[3] A. J. Atkinson, W. A. Colburn, V. G. DeGruttola et al., "Biomarkers and surrogate endpoints: preferred definitions and conceptual framework," Clinical Pharmacology and Therapeutics, vol. 69, no. 3, pp. 89-95, 2001.

[4] B. Sawitzki, A. Bushell, U. Steger et al., "Identification of gene markers for the prediction of allograft rejection or permanent acceptance," American Journal of Transplantation, vol. 7, no. 5, pp. 1091-1102, 2007.

[5] K. Christopher, T. F. Mueller, C. Ma, Y. Liang, and D. L. Perkins, "Analysis of the innate and adaptive phases of allograft rejection by cluster analysis of transcriptional profiles," Journal of Immunology, vol. 169, no. 1, pp. 522-530, 2002.

[6] A. Tefferi, M. E. Bolander, S. M. Ansell, E. D. Wieben, and T. C. Spelsberg, "Primer on medical genomics. Part III: microarray experiments and data analysis," Mayo Clinic Proceedings, vol. 77, no. 9, pp. 927-940, 2002.

[7] P. S. Hayden, A. El-Meanawy, J. R. Schelling, and J. R. Sedor, "DNA expression analysis: serial analysis of gene expression, microarrays and kidney disease," Current Opinion in Nephrology and Hypertension, vol. 12, no. 4, pp. 407-414, 2003.

[8] A. Henger, H. Schmid, and M. Kretzler, "Gene expression analysis of human renal biopsies: recent developments towards molecular diagnosis of kidney disease," Current Opinion in Nephrology and Hypertension, vol. 13, no. 3, pp. 313-318, 2004.

[9] S. Kurian, Y. Grigoryev, S. Head, D. Campbell, T. Mondala, and D. R. Salomon, "Applying genomics to organ transplantation medicine in both discovery and validation of biomarkers," International Immunopharmacology, vol. 7, no. 14, pp. 19481960, 2007.

[10] S. E. Ong and M. Mann, "Mass spectrometry-based proteomics turns quantitative," Nature chemical biology, vol. 1, no. 5, pp. 252-262, 2005.

[11] J. Bermúdez-Crespo and J. L. López, "A better understanding of molecular mechanisms underlying human disease," Proteomics Clinical Applications, vol. 1, no. 9, pp. 983-1003, 2007.

[12] R. A. Irizarry, B. Hobbs, F. Collin et al., "Exploration, normalization, and summaries of high density oligonucleotide array probe level data," Biostatistics, vol. 4, no. 2, pp. 249-264, 2003.

[13] G. K. Smyth, J. Michaud, and H. S. Scott, "Use of withinarray replicate spots for assessing differential expression in microarray experiments," Bioinformatics, vol. 21, no. 9, pp. 2067-2075, 2005.

[14] R. C. Gentleman, V. J. Carey, D. M. Bates et al., "Bioconductor: open software development for computational biology and bioinformatics," Genome biology, vol. 5, no. 10, p. R80, 2004.

[15] M. Sarwal, M. S. Chua, N. Kambham et al., "Molecular heterogeneity in acute renal allograft rejection identified by DNA microarray profiling," New England Journal of Medicine, vol. 349, no. 2, pp. 125-138, 2003.

[16] G. Einecke, J. Reeve, M. Mengel et al., "Expression of B cell and immunoglobulin transcripts is a feature of inflammation in late allografts," American Journal of Transplantation, vol. 8, no. 7, pp. 1434-1443, 2008.

[17] K. S. Famulski, G. Broderick, G. Einecke et al., "Transcriptome analysis reveals heterogeneity in the injury response of kidney transplants," American Journal of Transplantation, vol. 7, no. 11, pp. 2483-2495, 2007.
[18] T. F. Mueller, G. Einecke, J. Reeve et al., "Microarray analysis of rejection in human kidney transplants using pathogenesisbased transcript sets," American Journal of Transplantation, vol. 7, no. 12, pp. 2712-2722, 2007.

[19] E. C. Aquino-Dias, G. Joelsons, D. M. Da Silva et al., "Noninvasive diagnosis of acute rejection in kidney transplants with delayed graft function," Kidney International, vol. 73, no. 7, pp. 877-884, 2008.

[20] E. Marrer and F. Dieterle, "Promises of biomarkers in drug development-a reality check," Chemical Biology and Drug Design, vol. 69, no. 6, pp. 381-394, 2007.

[21] S. M. Flechner, S. M. Kurian, S. R. Head et al., "Kidney transplant rejection and tissue injury by gene profiling of biopsies and peripheral blood lymphocytes," American Journal of Transplantation, vol. 4, no. 9, pp. 1475-1489, 2004.

[22] S. Brouard, E. Mansfield, C. Braud et al., "Identification of a peripheral blood transcriptional biomarker panel associated with operational renal allograft tolerance," Proceedings of the National Academy of Sciences of the United States of America, vol. 104, no. 39, pp. 15448-15453, 2007.

[23] O. P. Günther, R. F. Balshaw, A. Scherer et al., "Functional genomic analysis of peripheral blood during early acute renal allograft rejection," Transplantation, vol. 88, no. 7, pp. 942951, 2009.

[24] G. V. Cohen Freue, M. Sasaki, A. Meredith et al., "Proteomic signatures in plasma during early acute renal allograft rejection," Molecular and Cellular Proteomics, vol. 9, no. 9, pp. 1954-1967, 2010.

[25] M. Martínez-Llordella, J. J. Lozano, I. Puig-Pey et al., "Using transcriptional profiling to develop a diagnostic test of operational tolerance in liver transplant recipients," Journal of Clinical Investigation, vol. 118, no. 8, pp. 2845-2857, 2008.

[26] M. Hernandez-Fuentes, B. Sawitzki, E. Perucha et al., "Identification of immune tolerance in renal transplants," American Journal of Transplantation, vol. 8, supplement 2, p. 292, 2008.

[27] K. Newell, A. Asare, T. Gisler et al., "A unique B cell signature associated with operational tolerance," American Journal of Transplantation, vol. 8, supplement 2, p. 316, 2008.

[28] O. Viklicky, P. Hribova, H. D. Volk et al., "Molecular phenotypes of acute rejection predict kidney graft prognosis," Journal of the American Society of Nephrology, vol. 21, no. 1, pp. 173-180, 2010.

[29] J. Ashton-Chess, E. Dugast, R. B. Colvin et al., "Regulatory, effector, and cytotoxic $\mathrm{T}$ cell profiles in long-term kidney transplant patients," Journal of the American Society of Nephrology, vol. 20, no. 5, pp. 1113-1122, 2009.

[30] J. Ashton-Chess, M. Giral, M. Mengel et al., "Tribbles-1 as a novel biomarker of chronic antibody-mediated rejection," Journal of the American Society of Nephrology, vol. 19, no. 6, pp. 1116-1127, 2008.

[31] Y. Matsui, A. Saiura, Y. Sugawara et al., "Identification of gene expression profile in tolerizing murine cardiac allograft by costimulatory blockade," Physiological Genomics, vol. 15, pp. 199-208, 2004.

[32] S. P. Cobbold, K. F. Nolan, L. Graca et al., "Regulatory T cells and dendritic cells in transplantation tolerance: molecular markers and mechanisms," Immunological Reviews, vol. 196, pp. 109-124, 2003.

[33] D. Zelenika, E. Adams, S. Humm et al., "Regulatory T cells overexpress a subset of Th2 gene transcripts," Journal of Immunology, vol. 168, no. 3, pp. 1069-1079, 2002.

[34] M. Sarwal, M. S. Chua, N. Kambham et al., "Molecular heterogeneity in acute renal allograft rejection identified by 
DNA microarray profiling," New England Journal of Medicine, vol. 349, no. 2, pp. 125-138, 2003.

[35] S. M. Flechner, S. M. Kurian, S. R. Head et al., "Kidney transplant rejection and tissue injury by gene profiling of biopsies and peripheral blood lymphocytes," American Journal of Transplantation, vol. 4, no. 9, pp. 1475-1489, 2004.

[36] V. Mas, D. Maluf, K. Archer et al., "Establishing the molecular pathways involved in chronic allograft nephropathy for testing new noninvasive diagnostic markers," Transplantation, vol. 83, no. 4, pp. 448-457, 2007.

[37] S. Brouard, E. Mansfield, C. Braud et al., "Identification of a peripheral blood transcriptional biomarker panel associated with operational renal allograft tolerance," Proceedings of the National Academy of Sciences of the United States of America, vol. 104, no. 39, pp. 15448-15453, 2007.

[38] M. Martínez-Llordella, J. J. Lozano, I. Puig-Pey et al., "Using transcriptional profiling to develop a diagnostic test of operational tolerance in liver transplant recipients," Journal of Clinical Investigation, vol. 118, no. 8, pp. 2845-2857, 2008.

[39] X. Xu, J. A. Golden, G. Dolganov et al., "Transcript signatures of lymphocytic bronchitis in lung allograft biopsy specimens," Journal of Heart and Lung Transplantation, vol. 24, no. 8, pp. 1055-1066, 2005.

[40] M. C. Deng, H. J. Eisen, M. R. Mehra et al., "Noninvasive discrimination of rejection in cardiac allograft recipients using gene expression profiling," American Journal of Transplantation, vol. 6, no. 1, pp. 150-160, 2006.

[41] G. Bodonyi-Kovacs, P. Putheti, M. Marino et al., "Gene expression profiling of the donor kidney at the time of transplantation predicts clinical outcomes 2 years after transplantation," Human Immunology, vol. 71, no. 5, pp. 451-455, 2010.

[42] K. Solez, R. B. Colvin, L. C. Racusen et al., "Banff 07 classification of renal allograft pathology: updates and future directions," American Journal of Transplantation, vol. 8, no. 4, pp. 753-760, 2008. 


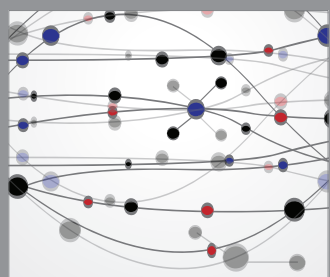

The Scientific World Journal
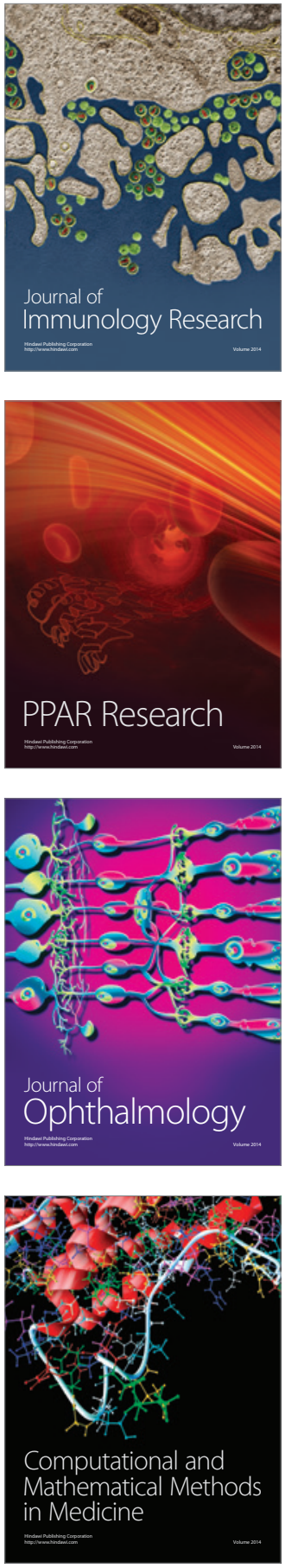

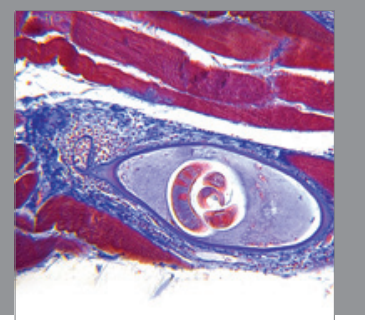

Gastroenterology

Research and Practice
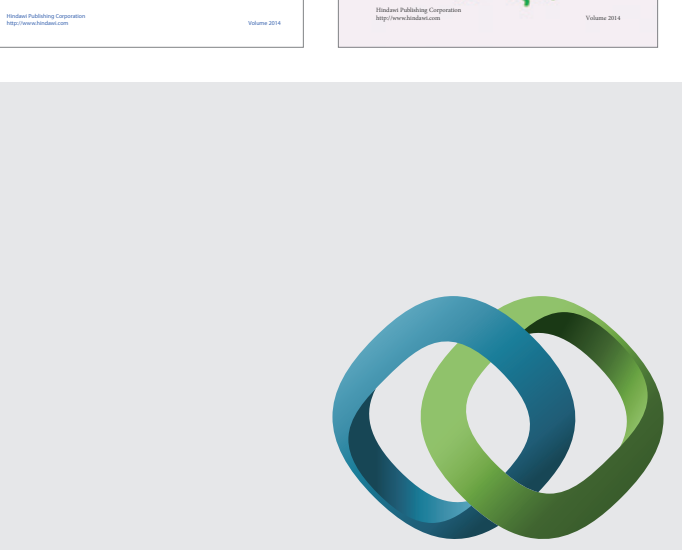

\section{Hindawi}

Submit your manuscripts at

http://www.hindawi.com
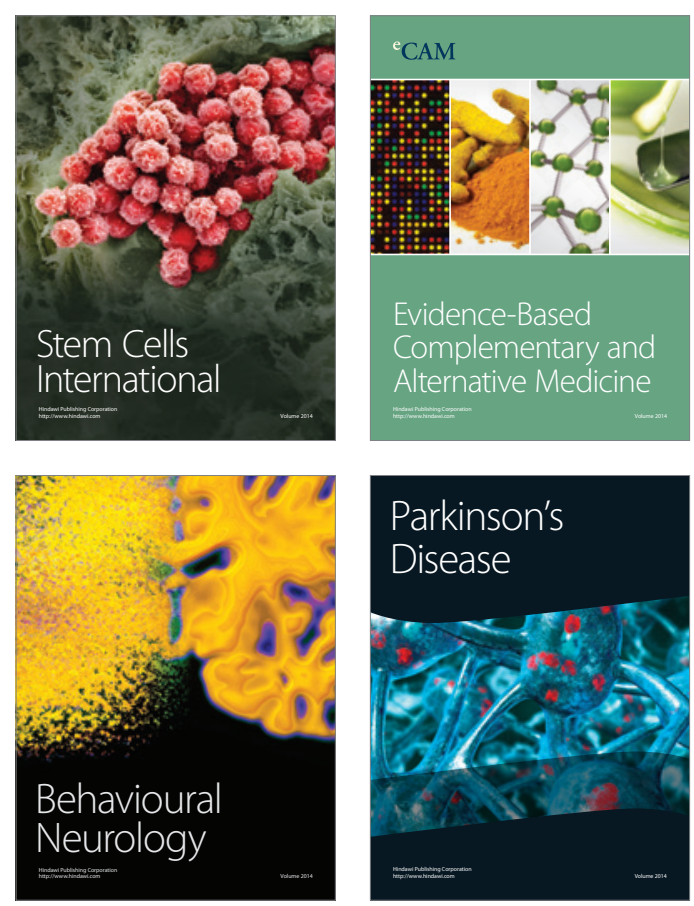

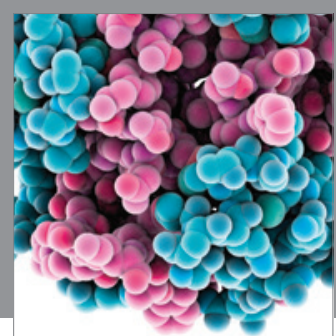

Journal of
Diabetes Research

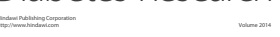

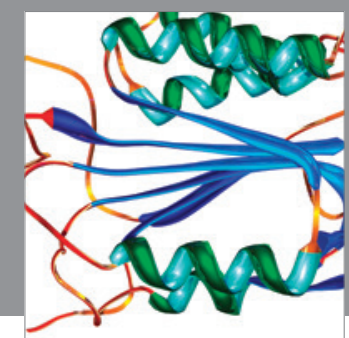

Disease Markers
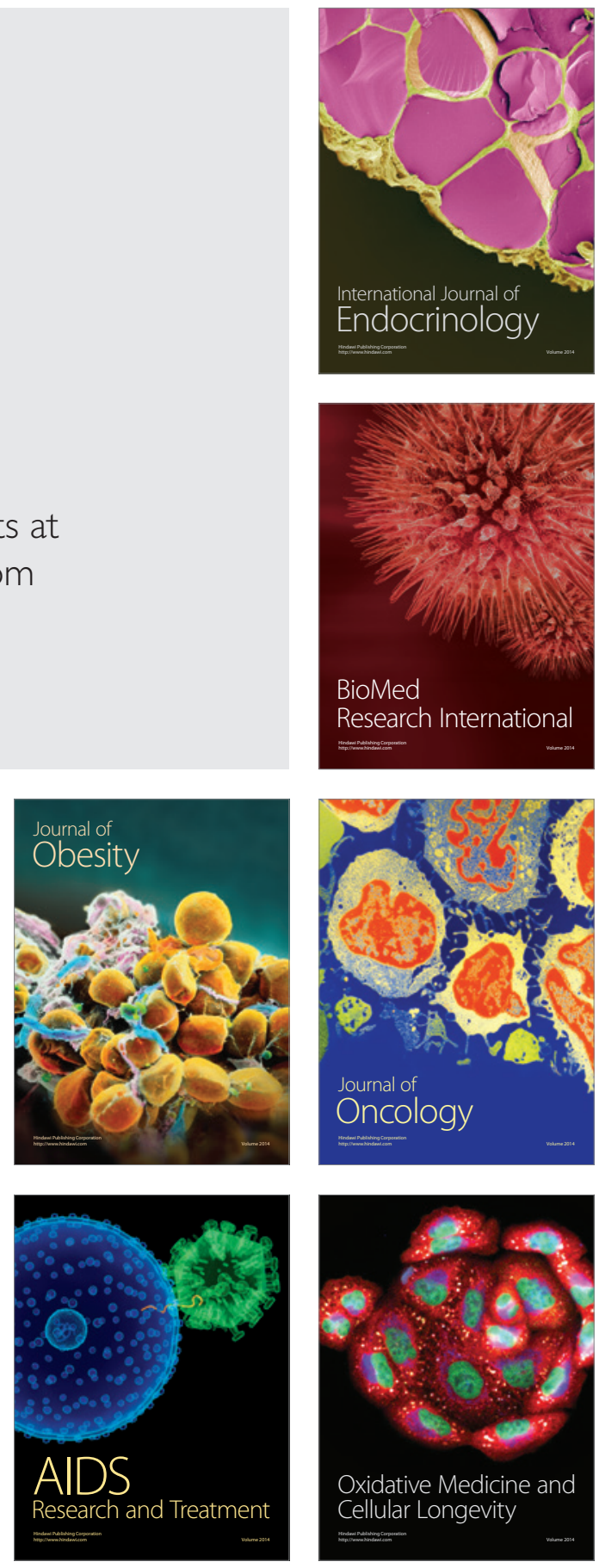